\title{
Illustrated larval key to lepidopteran pests of rice in Malaysia
}

\begin{abstract}
Here we provide an illustrated key to lepidopteran larvae that occur as pests on rice (Oryza) in Malaysia. We are unaware of a published key for this region for this vital commercial crop, and hence provide one based on easily observable features that could be useful for identification, early detection, and pest management by specialists and non-specialists alike (see discussion in Mukerji \& Singh 1951, Sri et al. 2010, Timm et al. 2007, Tillmon et al. 2000, Wagener et al. 2004).
\end{abstract}

Keyword: Lepidoptera 
